\title{
Patrones de susceptibilidad antimicrobiana de cepas de Shigella sonnei aisladas durante tres períodos diferentes en la Región Metropolitana, Chile
}

\author{
Alicia Marcoleta, Cecilia Toro, Valeria Prado, Marcela Serrano, \\ Pilar Fernández, Dona Benadof, Rossana Camponovo, Verónica Campos, Lorena Porte, \\ Juanita Zamorano, Camila Ortega, Belén Urqueta y M. Teresa Ulloa
}

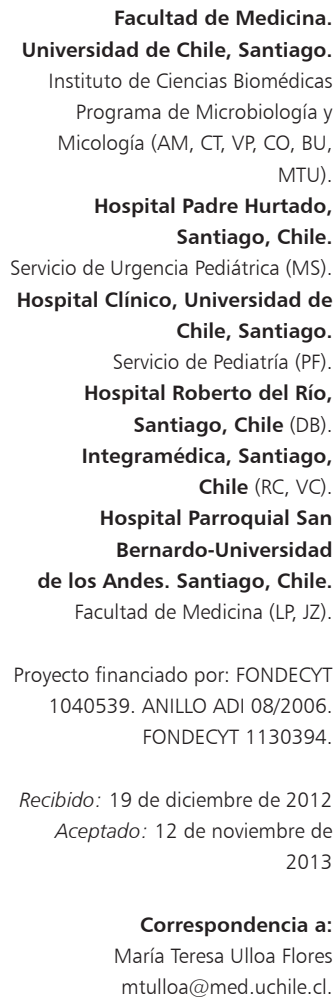

Facultad de Medicina. Universidad de Chile, Santiago. Instituto de Ciencias Biomédicas Programa de Microbiología y Micología (AM, CT, VP, CO, BU, MTU).

Hospital Padre Hurtado, Santiago, Chile. Servicio de Urgencia Pediátrica (MS). Hospital Clínico, Universidad de Chile, Santiago Servicio de Pediatría (PF). Hospital Roberto del Río,

Santiago, Chile (DB) Integramédica, Santiago Chile (RC, VC)

Hospital Parroquial San Bernardo-Universidad de los Andes. Santiago, Chile. Facultad de Medicina (LP, JZ)

Proyecto financiado por: FONDECYT 1040539. ANILLO ADI 08/2006. FONDECYT 1130394

Recibido: 19 de diciembre de 2012 Aceptado: 12 de noviembre de

\section{Antibiotic susceptibility patterns among Shigella sonnei, isolated during three different periods in Región Metropolitana, Chile}

Background: Shigella sonnei gastroenteritis improves clinically and microbiologically with antibacterial treatment; however choosing a useful drug is a universal challenge because of in vitro susceptibility of $S$. sonnei frequently evolves to be resistant. Objective: To evaluate in vitro susceptibility of $S$. sonnei strains isolated from patients attending at the Chilean Región Metropolitana and to know the evolution that resistant patterns of S. sonnei have experienced. Material: In this study, the antimicrobial susceptibility profile of 277 isolates of Shigella sonnei was compared. The analyzed periods of time were: period I (1995-1997) 85 strains; period II (2004-2006) 92 strains and period III (2008-2009) 100 strains, in Santiago, Chile. The method performed to analyze susceptibility patterns was the disc diffusion (Kirby-Bauer). Results: The strains showed rates of resistance to ampicillin: period I, 85.8\%; period II, 53.3\%; period III, 100\%, trimethoprim/sulfamethoxazole: period I, 50.5\%; period, II 46.7\%; period III, 100\%, chloramphenicol: period I, 36.4\%; period II, 12\%; period III, $100 \%$ and tetracycline: period I, $38.8 \%$; period II, $30.4 \%$; period III, $100 \%$. $98.9 \%$ of the strains showed susceptibility to quinolones. Significant differences were observed in patterns of antimicrobial resistance for both individuals and for multidrug resistance ( $\geq 3$ antimicrobials) in the three periods ( $\mathrm{p}<0.001, \chi 2$ test). Of all resistant strains, $17 \%$ were resistant to 1 or 2 antibiotics, while $65.7 \%$ showed a pattern of multidrug resistance; $100 \%$ of the period III strains presented multidrug resistance. Conclusion: These results showed the temporal resistance dynamics of $S$. sonnei circulating strains in the Chilean Región Metropolitana. Due to the endemic behavior of shigellosis in Chile, it is urgent to maintain permanent surveillance of antimicrobial resistance profiles to improve both prevention and treatment of shigellosis.

Key words: Shigella, shigellosis, antimicrobial resistance, multidrug resistance, diarrhea.

Palabras clave: Shigella, shigelosis, resistencia antimicrobiana, multi-resistencia, diarrea.

\section{Introducción}

L as infecciones entéricas que producen diarrea son un importante problema de salud pública en el mundo. En países en vías de desarrollo, la diarrea de origen infeccioso contribuye substancialmente a la malnutrición, resultando en alta morbilidad y mortalidad, particularmente en pediatría ${ }^{1}$. La shigelosis continúa siendo la enfermedad gastrointestinal más común en países desarrollados y en desarrollo ${ }^{2}$. La infección es transmitida vía fecal-oral a través del contacto directo persona-persona y en menor grado por contacto con agua, alimentos o fomites contaminados. La diseminación de la shigelosis se ve favorecida por la baja dosis infectante (10-500 unidades formadoras de colonias, ufc), por las malas condiciones higiénicas y en ciertos ambientes por la inadecuada eliminación de excretas ${ }^{3-5}$. Shigella sonnei corresponde a la especie aislada con mayor frecuencia en países desarrollados ${ }^{6}$. Sin embargo, actualmente ha emergido como un problema en países en vías de desarrollo, pareciendo reemplazar a $S$. flexneri ${ }^{2}$.

En base al antígeno $\mathrm{O}$ del lipopolisacárido (LPS), en Shigella se reconocen cuatro especies: $S$. dysenteriae (serogrupo A con 13 serotipos), S. flexneri (serogrupo B con 15 serotipos incluyendo subtipos), S. boydii (serogrupo C con 18 serotipos) y $S$. sonnei (serogrupo D con un serotipo). Shigella flexneri y $S$. sonnei son las especies prevalentes y endémicas en América Latina y en países industrializados ${ }^{7}$. En Chile, $S$. flexneri y $S$. sonnei, constituyen más de $80 \%$ de las cepas de Shigella aisladas, con una proporción similar entre ambas, predominando $S$. sonnei cada dos o tres años ${ }^{8-10}$. Investigaciones realizadas en niños chilenos, mostraron que un número reducido de serotipos (S. sonnei, S. flexneri $2 \mathrm{a}, S$. flex- 
neri 2 b y $S$. flexneri 6) son responsables de $90 \%$ de los episodios de shigelosis y que se mantienen relativamente estables en el tiempo ${ }^{10}$. En un informe emitido recientemente, el Instituto de Salud Pública de Chile (ISP) confirmó que $S$. sonnei es el serotipo prevalente en nuestro país con un incremento de $34 \%$ de cepas confirmadas durante el primer trimestre del año 2012, en comparación con igual período del año 2011 y de $68 \%$ respecto del año $2010^{11}$.

A pesar que la gastroenteritis causada por Shigella spp. es normalmente auto-limitada, se recomienda la terapia antimicrobiana, que ha demostrado ser eficiente en la reducción de la duración de la enfermedad, del riesgo letal y el período de excreción de Shigella luego que remiten los síntomas ${ }^{12-14}$. La resistencia antimicrobiana ha complicado el tratamiento de la shigelosis desde 1940, cuando se detectaron las primeras cepas de Shigella resistentes a sulfonamidas en Japón. Subsecuentemente, Shigella adquirió resistencia a tetraciclina y cloranfenicol durante la década de 1950, con lo cual ampicilina se convirtió en el fármaco de elección. A principios de 1980 aparecieron las cepas resistentes a ampicilina y se diseminaron en Asia y África. Posteriormente, cotrimoxazol se convirtió en el tratamiento de elección y siguió siendo efectivo hasta mediados de $1980^{15}$. Hasta el año 2007, las fluoroquinolonas, $\beta$-lactámicos y cotrimoxazol eran los antimicrobianos de elección para el tratamiento de la shigelosis. Sin embargo, su utilización nuevamente se vio restringida debido al surgimiento de cepas resistentes. La rápida emergencia de cepas multi-resistentes se debe en gran parte a su habilidad de adquirir y diseminar genes exógenos asociados con elementos genéticos móviles tales como plásmidos, transposones, integrones e islas genómicas ${ }^{16}$. Actualmente, existe un problema creciente universal respecto al aumento de resistencia antibacteriana de Shigella spp. ${ }^{12}$. Numerosos estudios internacionales han demostrado que la infección por Shigella multiresistente es de carácter generalizado y que puede ser adquirida durante viajes ${ }^{17}$. Es frecuente la resistencia de Shigella spp. a ampicilina y cotrimoxazol, que se han utilizado en pediatría durante las cuatro décadas pasadas ${ }^{13}$. Un panorama similar se ha observado en Chile, como lo indica un estudio de vigilancia que demostró que cerca de $50 \%$ de las cepas aisladas presentan resistencia a más de un antimicrobiano ${ }^{18}$. Estudios anteriores demostraron frecuencias de resistencia en cepas de Shigella para ampicilina (87\%), cotrimoxazol (76\%), tetraciclina (73\%) y cloranfenicol (52\%). Estas cifras coinciden con estudios internacionales, confirmando la globalización del proble$\mathrm{ma}^{10}$. Dado que el tratamiento con antimicrobianos reduce la transmisión, su utilización durante un brote puede representar una importante medida de salud pública. Con el aumento de la resistencia antibacteriana, el conocimiento de los perfiles de susceptibilidad antimicrobiana de cepas prevalentes resulta útil en la guía del tratamiento empírico en casos esporádicos ${ }^{17}$.

El objetivo de este estudio fue evaluar la susceptibilidad a antimicrobianos y comparar los patrones de resistencia de $S$. sonnei aisladas de cuadros clínicos en pacientes de la Región Metropolitana, Chile. Para esto, se analizaron cepas obtenidas de tres períodos, durante los años 1995-1997 (período I), 2004-2007 (período II) y 2008-2009 (período III), mediante método de difusión en agar, para establecer el comportamiento de este patógeno en nuestra realidad local y sugerir en base a datos actualizados, recomendaciones oportunas para la prevención y administración de terapia antibacteriana en los casos de shigelosis.

\section{Material y Métodos}

\section{Cepas}

Se estudiaron 277 cepas de $S$. sonnei aisladas de episodios diarreicos atendidos en la Región Metropolitana (RM) Norte (Consultorio de Colina, Hospital Roberto del Río, Hospital Clínico Universidad de Chile) RM Sur: H. Parroquial de San Bernardo, H. Padre Hurtado. RM Central: Integramédica, RM Oriente: Integramédica y RM Occidente: Integramédica.

De ellos, $87 \%$ corresponde a pacientes bajo 15 años de edad. Las muestras correspondieron a tres diferentes períodos: 85 cepas en el período I (1995-1997); 92 cepas en el período II (2004-2006) y 100 cepas en el período III (2008-2009).

\section{Estudio de susceptibilidad in vitro}

Se utilizó el test de difusión en agar para: ampicilina $(10 \mu \mathrm{g})$, amoxicilina/ácido clavulánico $(20 / 10 \mu \mathrm{g})$, trimetoprim/sulfametoxazol-cotrimoxazol (1,25/23,75 $\mu \mathrm{g})$, cloranfenicol $(30 \mu \mathrm{g})$, tetraciclina $(30 \mu \mathrm{g})$, ciprofloxacina $(5 \mu \mathrm{g})$ y ácido nalidíxico $(30 \mu \mathrm{g})$. Los resultados fueron interpretados según las normas vigentes CLSI $2012^{19}$.

\section{Análisis estadístico}

La significancia de las diferencias entre las proporciones de resistencia antimicrobiana y las prevalencias relativas de $S$. sonnei de los tres diferentes períodos, se determinaron mediante el test $\chi^{2}$ de Fisher. Se utilizó el software Statgraphics Plus versión 5.1 para obtener el p value y se consideró estadísticamente significativo un valor $\leq$ a 0,05 .

\section{Resultados}

Del total de 277 cepas $S$. sonnei analizadas, se pudo observar que $48(17,3 \%)$ cepas eran sensibles a todos los 


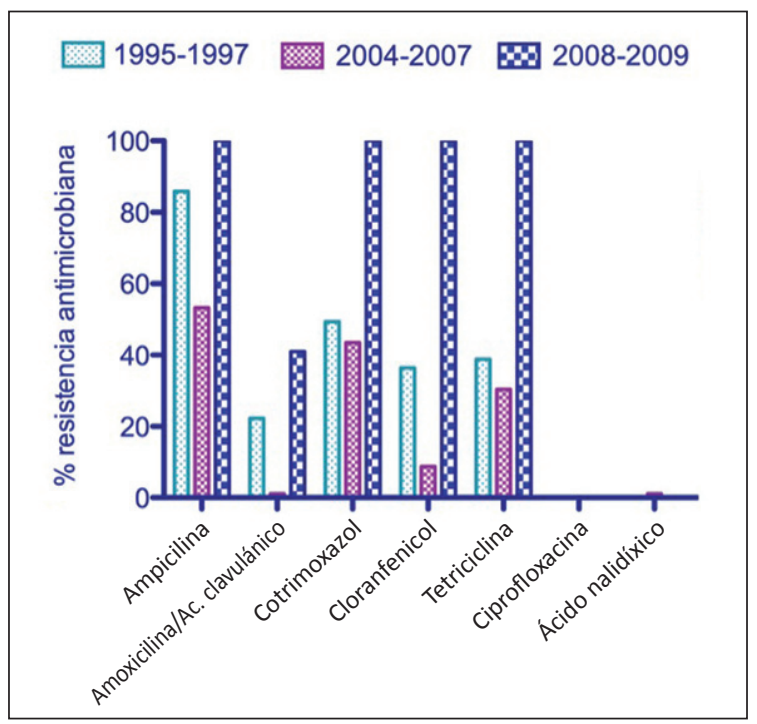

Figura 1. Perfiles de resistencia a antimicrobianos en cepas de $S$. sonnei, aisladas en la Región Metropolitana durante los períodos I: 1995-1997, II: 2004-2007 y III: 2008-2009.

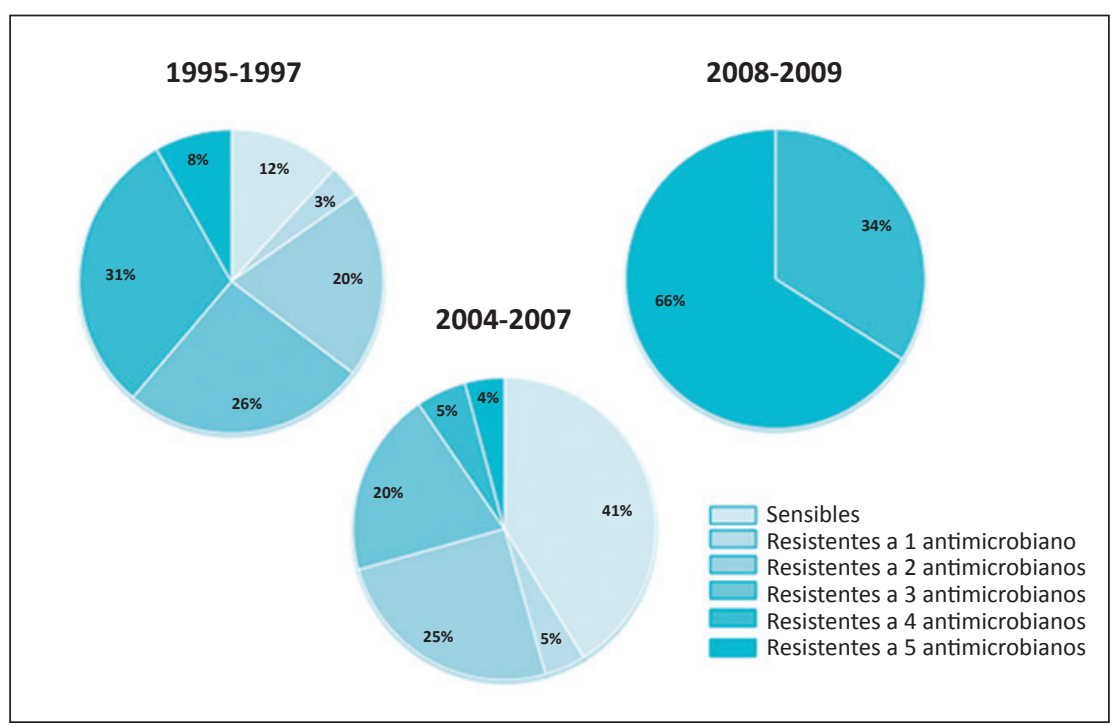

Figura 2. Patrones de multi-resistencia frente a cinco antimicrobianos en cepas de $S$. sonnei aisladas en la Región Metropolitana durante los períodos 1995-1997, 2004-2007 y 2008-2009.
Tabla 1. Distribución de patrones de resistencia entre 277 cepas de Shigella sonnei aisladas durante tres períodos en la Región Metropolitana, Chile

\begin{tabular}{|c|c|c|c|c|c|c|}
\hline & \multicolumn{2}{|c|}{$\begin{array}{c}\text { Período I } \\
(1995-1997) \\
n=85\end{array}$} & \multicolumn{2}{|c|}{$\begin{array}{c}\text { Período II } \\
(2004-2007) \\
\text { n }=92\end{array}$} & \multicolumn{2}{|c|}{$\begin{array}{c}\text { Período III } \\
(2008-2009) \\
n=100\end{array}$} \\
\hline & $\mathbf{n}$ & $\%$ & $n$ & $\%$ & $\mathrm{n}$ & $\%$ \\
\hline \multicolumn{7}{|l|}{ Susceptibles } \\
\hline AMP, SXT, AMC, CAF, TET, AN, CIP & 10 & 11,8 & 38 & 41,3 & 0 & 0 \\
\hline \multicolumn{7}{|l|}{ Resistentes a 1 antibacteriano } \\
\hline AMP & 3 & 3,5 & 2 & 2,2 & 0 & 0 \\
\hline SXT & 0 & 0 & 2 & 2,2 & 0 & 0 \\
\hline \multicolumn{7}{|l|}{ Resistentes a 2 antibacterianos } \\
\hline AMP, SXT & 15 & 17,6 & 19 & 20,7 & 0 & 0 \\
\hline AMP, AMC & 2 & 2,4 & 2 & 2,2 & 0 & 0 \\
\hline AN, TET & 0 & 0 & 1 & 1,1 & 0 & 0 \\
\hline CAF, TET & 0 & 0 & 1 & 1,1 & 0 & 0 \\
\hline \multicolumn{7}{|l|}{ Resistentes a 3 antibacterianos } \\
\hline AMP, CAF, TET & 1 & 1,2 & 1 & 1,1 & 0 & 0 \\
\hline AMP, SXT, TET & 1 & 1,2 & 16 & 17,4 & 0 & 0 \\
\hline AMP, AMC, SXT & 20 & 23,5 & 1 & 1,1 & 0 & 0 \\
\hline \multicolumn{7}{|l|}{ Resistentes a 4 antibacterianos } \\
\hline AMP, SXT, CAF, TET & 0 & 0 & 1 & 1,1 & 34 & 34 \\
\hline AMP, AMC, SXT, TET & 1 & 1,2 & 0 & 0 & 0 & 0 \\
\hline AMP, AMC, CAF, TET & 25 & 29,4 & 4 & 4,3 & 0 & 0 \\
\hline \multicolumn{7}{|l|}{ Resistentes a 5 antibacterianos } \\
\hline AMP, AMC, SXT, CAF, TET & 7 & 8,2 & 4 & 4,2 & 66 & 66 \\
\hline
\end{tabular}

*AMP: ampicilina, SXT: cotrimoxazol, AMC: amoxicilina/ácido clavulánico, CAF: cloranfenicol, TET: tetraciclina, AN: ácido nalidíxico, CIP: ciprofloxacina. antimicrobianos estudiados y $229(82,7 \%)$ presentaron resistencia a uno o más antibacteriano: 47 (17\%) presentaron resistencia a uno o dos antibacterianos y 182 $(65,7 \%)$ fueron multi-resistentes ( $p<0,001$; Test de $\chi^{2}$ ).

Los porcentajes de resistencia antimicrobiana durante los tres períodos fueron: ampicilina: período I, 85,8\%; período II, $53,3 \%$; período III, $100 \%$, cotrimoxazol: período I, 50,5\%; período, II 46,7\%; período III, $100 \%$, cloranfenicol: período I, $36,4 \%$; período II, $12 \%$; período III, $100 \%$ y tetraciclina: período I, $38,8 \%$; período II, $30,4 \%$; período III, $100 \%$. (Figura 1). Además, se detectó una mínima resistencia a quinolonas (ciprofloxacina, ácido nalidíxico), con $1,1 \%$ de resistencia a ácido nalidíxico, sólo en el período II. Es importante destacar que el período con menor porcentaje de resistencia fue el correspondiente a los años 2004-2007. Estas diferencias son significativas para cada antimicrobiano individual en los tres períodos $\left(\mathrm{p}<0,001\right.$; Test de $\left.\chi^{2}\right)$.

Se encontraron además nueve patrones de resistencia en el período I, 12 en el período II y sólo dos en el período III (Tabla 1). Los porcentajes de resistencia más altos correspondieron a las combinatorias* AMP/SXT (período I: $17,6 \%$; período II: 20,7\%), AMP/AMC/SXT (período I: 23,5\%), AMP/SXT/TET (período II: 17,4\%), AMP/AMC/CAF/TET (período I: 29,4\%), AMP/SXT/ CAF/TET (período III: 34\%), AMP/AMC/SXT/CAF/ TET (período III: 66\%). Posteriormente, los resultados se analizaron según patrones de multi-resistencia en los distintos períodos, considerando las combinatorias entre

\footnotetext{
*Significado de abreviaturas en la Tabla 1.
} 
ampicilina, cotrimoxazol, amoxicilina/ácido clavulánico, cloranfenicol y tetraciclina (Tabla 1). Se definió como cepa multi-resistente a aquella cepa resistente a tres o más antimicrobianos ${ }^{23}$. Según el patrón de multi-resistencia, observamos importantes diferencias entre los tres períodos: $100 \%$ de las cepas del período III presentaron multi-resistencia en comparación a los períodos I $(64,7 \%)$ y II $(29,3 \%), p<0,0001$ (Figura 2).

\section{Discusión}

La resistencia antimicrobiana se ha extendido más allá del recinto hospitalario, transformándose en un problema de la comunidad. Las cepas resistentes pueden rastrearse desde la comunidad al hospital y viceversa, lo cual indica que la resistencia antimicrobiana es un fenómeno más transversal ${ }^{20}$. La terapia antimicrobiana es una herramienta valiosa en el control de la shigelosis, ya que reduce la duración, la intensidad de la enfermedad y previene complicaciones, eventualmente letales. Desafortunadamente, a partir de las décadas pasadas se han seleccionado cepas de Shigella spp. resistentes a los antimicrobianos de uso común. En parte, dicha resistencia se debe a que hasta antes del año 2000, no se habían descubierto nuevas clases de antimicrobianos, siendo utilizados desde 1960 sólo antimicrobianos con pequeñas modificaciones ${ }^{16}$. La selección y diseminación de la resistencia antimicrobiana entre las especies de Shigella es un problema creciente de salud pública, que complica el manejo terapéutico de casos graves de shigelosis. Estudios realizados en diferentes regiones de Chile han reportado un aumento en el aislamiento de Shigella resistente o multi-resistente a antimicrobianos de uso común y clínicamente importantes ${ }^{21}$.

Con respecto a los serotipos circulantes asociados a los episodios de shigelosis en nuestro país, se ha establecido que tanto $S$. sonnei como $S$. flexneri 2a fueron responsables de aproximadamente $95 \%$ de los casos detectados en el período correspondiente a los años 2004-2005 ${ }^{10}$. Sin embargo, se ha observado una clara prevalencia de $S$. sonnei en períodos posteriores ${ }^{22}$. De hecho, durante el verano del año 2009 se registró un brote de shigelosis en el país, cuyo agente etiológico fue $S$. sonnei. Cepas pertenecientes a este brote se encuentran incluidas en este estudio. En el período 2010, S. sonnei se mantuvo como la especie más frecuente $(61 \%)$, particularmente en el grupo etario entre 2 y 20 años. Los casos de shigelosis reportados en el año 2011 y 2012 también se asociaron principalmente con $S$. sonnei. Sin embargo, no se dispone de información de susceptibilidad antimicrobiana para estas cepas ${ }^{11}$.

Si bien es cierto, los grupos analizados en nuestro estudio no son homogéneos respecto al periodo de tiempo de obtención de las cepas, cabe destacar que la compa- ración se establece principalmente entre cepas obtenidas durante los periodos de verano, lo cual corresponde al comportamiento estacional que presentan los síndromes diarreicos por este agente. Aún cuando las cepas del grupo III fueron aisladas en el periodo de verano 2008-2009, cuando se produjo un brote nacional, este grupo está constituido por muestras representativas de las diversas áreas de la Región Metropolitana, lo cual minimiza en parte, la probabilidad de falta de representatividad de las cepas incluidas en nuestro estudio.

Haciendo un análisis global de las cepas evaluadas en este trabajo (n: 277), 17,3\% fue sensible a todos los antimicrobianos estudiados y $82,7 \%$ mostró resistencia a uno o más antibacterianos. Del total de las cepas resistentes, $17 \%$ presentó resistencia a uno o dos antibacterianos, mientras que $65,7 \%$ mostró un patrón de multi-resistencia. Con respecto a los diferentes patrones de resistencia a antimicrobianos de $S$. sonnei encontrados en los tres períodos (1995-1997; 2004-2007; 2008-2009), se pudo observar que éstos no siguieron un comportamiento estable en el tiempo; mientras la totalidad de cepas en el período III mostró un patrón de multi-resistencia, en el período II éste alcanzó sólo a 29,3\%.

Dicho fenómeno puede evidenciar la lenta penetración de la resistencia antimicrobiana adquirida en la comunidad, sumado a que cada individuo actúa como reservorio de bacterias resistentes. Por otro lado, se ha observado que al administrar los mismos antimicrobianos a la población durante años, se impide el efecto de recolonización del nicho por parte de competidores naturales susceptibles $^{20}$. El elevado y constante porcentaje de resistencia a ampicilina observado en las cepas $(80,1 \%)$ podría ser explicado por el amplio uso de $\beta$-lactámicos para tratar diversas infecciones en niños chilenos, lo cual originaría una selección de cepas resistentes y propagación de genes de resistencia a $\beta$-lactámicos en la microbiota normal intestinal que pueden transmitirse fácilmente entre Enterobacteriaceae comensales y enteropatógenas, tanto in vivo como in vitro ${ }^{21}$. Actualmente, en países desarrollados, las fluoroquinolonas constituyen el antimicrobiano de elección para el tratamiento de la shigelosis en adultos. En cambio, en niños con episodios más intensos, puede considerarse el uso de azitromicina o un tratamiento breve con quinolonas. No obstante, en regiones como nuestro país, donde la shigelosis tiene carácter endémico, las recomendaciones respecto a la selección de antimicrobianos deben ser periódicamente actualizadas según los patrones locales de resistencia antibacteriana de Shigella. Esto, sumado a variaciones en la prevalencia de serogrupos entre cepas de Shigella, genera una mayor dificultad para seleccionar un antimicrobiano apropiado en el tratamiento de la shigelosis ${ }^{15}$.

Los resultados de nuestro estudio demuestran que los patrones de resistencia a antimicrobianos en Shigella no 
presentan un comportamiento predecible; por ende, se debe realizar un uso más prudente de los actuales antimicrobianos, limitándose a los casos graves de manera que se favorezca la disminución de la resistencia y con esto, el resurgimiento de la utilidad de los antimicrobianos de uso común como terapia efectiva.

Además, es necesario recalcar la importancia de mantener los programas de vigilancia epidemiológica, que permiten disponer de información actualizada sobre los serotipos prevalentes y los patrones de resistencia antimicrobiana en las cepas circulantes de Shigella spp. en nuestro país, para así lograr implementar medidas preventivas y terapéuticas adecuadas.

Agradecimientos: A Verónica Iglesias, Escuela de Salud Pública de la Universidad de Chile, por su apoyo en el análisis estadístico y a Luisa Núñez S. por apoyo técnico.

\section{Resumen}

Introducción: La infección entérica producida por Shigella sonnei mejora clínicamente y microbiológicamente con antibioterapia; sin embargo, la elección del antimicrobiano es un problema universal pues la susceptibilidad in vitro de $S$. sonnei evoluciona frecuentemente hacia la resistencia. Objetivo: Evaluar la susceptibilidad in vitro a antimicrobianos de $S$. sonnei y conocer la evolución que han experimentado los patrones de resistencia de cepas aisladas de cuadros clínicos en pacientes de la Región Metropolitana, Chile. Material y Métodos: Se comparó el perfil de susceptibilidad a antimicrobianos, de 277 cepas clínicas de $S$. sonnei aisladas durante tres períodos: período I (1995-1997) 85 cepas; período II (2004-2006) 92 cepas y período III (2008-2009) 100 cepas, en Santiago, Chile. El perfil de susceptibilidad a antimicrobianos se determinó mediante test de difusión en agar. Resultados: Las tasas de resistencia de las cepas en los periodos I, II y III respectivamente fueron: ampicilina: $85,8 \% ; 53,3 \%$; $100 \%$, cotrimoxazol: $50,5 \% ; 46,7 \% ; 100 \%$, cloranfenicol: $36,4 \% ; 12 \% ; 100 \%$ y tetraciclina: $38,8 \% ; 30,4 \% ; 100 \%$. El $98,9 \%$ de las cepas fue susceptible a quinolonas. Se observó diferencias significativas en los porcentajes de resistencia para antimicrobianos individuales y multiresistencia ( $\geq 3$ antimicrobianos) en los tres períodos $(\mathrm{p}<0,001$; Test de $\chi 2)$. De las cepas resistentes, 17\% presentó resistencia a uno ó dos antimicrobianos, $65,7 \%$ mostró multi-resistencia antimicrobiana. El 100\% de las cepas del período III presentó multi-resistencia. Discusión: Estos resultados evidencian la dinámica temporal de la resistencia en cepas de $S$. sonnei circulantes en la Región Metropolitana. Dado que en Chile la shigelosis tiene un carácter endémico, es prioritario mantener una vigilancia constante de los perfiles de resistencia a antimicrobianos, para mejorar la prevención y el tratamiento de la shigelosis.

\section{Referencias bibliográficas}

1.- Navaneethan U, Giannella R A. Mechanisms of infectious diarrhea. Nat Clin Pract Gastroenterol Hepatol 2008; 5: 637-47.

2.- Holt K E, Baker S, Weill F-X, Holmes E C, Kitchen A, Yu J, Sangal V, et al. Shigella sonnei genome sequencing and phylogenetic analysis indicate recent global dissemination from Europe. Nature Genetics 2012; 44: 1056-9.

3.- Mahendra H K, Uma S B. Infective dose of foodborne pathogens in volunteers: a review. $\mathrm{J}$ Food Safety 2001; 21: 49-73.

4.- Niyogi S K, Shigellosis. J Microbiol 2005; 43: 133-43.

5.- Chompook P, Samosornsuk S, von Seidlein L, Jitsanguansuk S, Sirima N, Sudjai, et al. Estimating the burden of shigellosis in Thailand: 36-month population-based surveillance study. Bull World Health Organ 2005; 83: 739-46.

6.- Boumghar-Bourtchai L, Mariani-Kurkdjian P, Bingen E, Filliol I \& Dhalluin A. Macrolideresistant Shigella sonnei. Emerg Infect Dis 2008; 14: 1297-9.

7.- Kotloff K L, Winickoff J P, Ivanoff B, Clemens J D, Swerdlow D L, Sansonetti P J, et al. Global burden of Shigella infections: implications for vaccine development and implementation of control strategies. Bull World Health Organ 1999; 77: 651-66.

8.- Carrasco S, Solari V, Prado V, Suazo L, Arellano C, Hernández M, et al. Brote de shigelosis en una escuela de educación básica. Rev Chilena Infectol 2000; 17: 122-8.

9.- Delpiano L, Tejerina H, Cona E, Aviles C. Patrones de susceptibilidad in vitro y comportamiento clínico de Shigella. Rev Chilena Infectol 2001; 18: 101-7.

10.- Hamilton-West C, Prado V, Hormazábal J C, Lagos R, Benadof Z D, Mendoza C, et al. Epidemiología clínica y molecular de las infecciones por Shigella spp en niños de la Región Metropolitana durante el verano 2004-2005. Rev Med Chile 2007; 135: 1388-96.

11.- Instituto de Salud Pública de Chile (ISP) Informe de resultados de laboratorio Shigella spp. 2010-2012. 2012; p1-10. http://www.ispch. cl/sites/default/files/Vigilancia_Shigella_spp_0. pdf

12.- Ashkenazi S, Levy I, Kazaronovski V, Samra Z. Growing antimicrobial resistance of Shigella isolates. J. Antimicrob. Chemother 2003; 51: 427-9.
13.- Ghosh S, Pazhani G P, Chowdhury G, Guin S, Dutta S, Rajendran K, et al. Genetic characteristics and changing antimicrobial resistance among Shigella spp. isolated from hospitalized diarrhoeal patients in Kolkata, India. J Med Microbiol 2011; 60: 1460-6.

14.- Haukka \& Siitonen. Emerging resistance to newer antimicrobial agents among Shigella isolated from Finnish foreign travellers. Epidemiol. Infect 2008; 136: 476-82.

15.- Niyogi S K. Increasing antimicrobial resistance an emerging problem in the treatment of shigellosis. Clin Microbiol Infect 2007; 13: 1141-3.

16.- Vrints M, Mairiaux E, Van Meervenne E, Collard J-M, Bertrand S. Surveillance of antibiotic susceptibility patterns among Shigella sonnei strains isolated in Belgium during the 18-year period 1990 to 2007. J Clin Microbiol 2009; 47: 1379-85.

17.- Shiferaw B, Solghan S, Palmer A, Joyce K, Barzilay E J, Krueger A, et al. Antimicrobial susceptibility patterns of Shigella isolates in Foodborne Diseases Active Surveillance Network (FoodNet) sites, 2000-2010. Clin Infect Dis 2012; 54: S458-63.

18.- Pidal P, Prado V, Trucco O, Valdivieso F, 
Díaz M, Ojeda A, Grupo PRONARES.

Panorama de la resistencia antimicrobiana de Shigella sp. en 10 hospitales chilenos Proyecto Pronares. Rev Panam Infectol 1999; Supl 1: S-19.

19.- CLSI 2012. Performance Standards for Antimicrobial Susceptibility Testing Twenty Second Informational Supplement. M100, 2012; $\mathrm{S} 22$, vol $32 \mathrm{~N}^{\circ} 3$.
20.- Levy S B, Marshall B. Antibacterial resistance worldwide: causes, challenges and responses. Nature Medicine 2004; 10: S122-9.

21.- Fullá N, Prado V, Durán C, Lagos R, Levine M M. Surveillance for antimicrobial resistance profiles among Shigella species isolated from a semirural community in the northern administrative area of Santiago, Chile. Am J Trop Med Hyg 2005; 72: 851-4.
22.- Alerte V, Cortés A S, Díaz T J, Vollaire Z J, Espinoza M ME, Solari G, et al. Brotes de enfermedades transmitidas por alimentos y agua en la Región Metropolitana, Chile (2005-2010). Rev Chilena Infectol 2012; 29: 26-31.

23.- Zhang W, Luo Y, Li J, Lin L, Ma Y, Hu C, et al. Wide dissemination of multidrug-resistant Shigella isolates in China. J Antimicrob Chemother 2011; 66: 2527-35. 